\title{
An Iterative Modal Identification Algorithm for Structural Health Monitoring Using Wireless Sensor Networks
}

\author{
Siavash Dorvash, ${ }^{\text {a) }}$ Shamim N. Pakzad, ${ }^{\text {b) }}$ and Liang Cheng ${ }^{\mathrm{c})}$
}

\begin{abstract}
A novel modal identification approach for the use of a wireless sensor network (WSN) for structural health monitoring is presented, in which the computational task is distributed among remote nodes to reduce the communication burden of the network and, as a result, optimize the time and energy consumption of the monitoring system. Considering the need for having an agile system to capture the earthquake response and also the limited energy resource in WSN, such algorithms for speeding the analysis time and preserving energy are essential. The algorithm of this study, called iterative modal identification (IMID), relies on an iterative estimation method that solves for unknown parameters in the absence of complete information about the system. Applying IMID in WSN-based monitoring systems results in significant savings in time and energy. Validation through implementation of the algorithm on numerically simulated and experimental data illustrates the superior performance of this approach.
\end{abstract} [DOI: $10.1193 / 1.4000133]$

\section{INTRODUCTION}

Structural health monitoring (SHM) techniques have been the focus of much research in recent years and have significantly improved by incorporating advancements in sensing and communication technology. In the new generation of SHM systems, attention is turned to the scalability of the sensing network (Liu et al. 2003, Swartz et al. 2005, Pakzad et al. 2008). The hardware components for these scalable networks can be achieved by utilizing wireless sensor networks (WSNs). A WSN integrates a base station and remote sensors, with computational capability, communicating with each other through radio signals. Reduction in the instrumentation cost and labor, together with the capability of on-board processing at sensing nodes, are critical enhancements, brought to SHM systems by using WSN. Over the past few years, researchers have developed and deployed different WSN platforms for SHM and shown the potential that exists in application of WSN in the health monitoring of infrastructure (Tanner et al. 2003, Lynch et al. 2004a, Chung et al. 2004, Lynch et al. 2005, Pakzad et al. 2008, Whelan and Janoyan 2009, Pakzad 2010, Kim et al. 2010, Cho et al. 2010, Jang et al. 2010). While WSN makes the deployment of SHM more convenient, a few challenges have restricted its application in large-scale and long-term health monitoring of structural systems. Major challenges in application of WSN are the latency in the process due to

\footnotetext{
a) Ph.D. candidate, Department of Civil and Environmental Engineering, Lehigh University, Bethlehem, PA

b) Assistant Professor, Department of Civil and Environmental Engineering, Lehigh University, Bethlehem, PA

c) Associate Professor, Department of Computer Science and Engineering, Lehigh University, Bethlehem, PA
} 
the low data bandwidth and the difficulties in providing operational power of sensors for long-term monitoring. Particularly in the event of an earthquake, WSNs need to be responsive in capturing and processing the data. Thus, new approaches are required to address these challenges and make the existing WSN technology suitable for a wide variety of applications.

In recent years, different approaches have been developed to address the power consumption issue. Energy harvesting (Grisso et al. 2005), wireless power transmission (Das et al. 1998), and optimal power usage strategy are some examples. While these methods rely on technological developments, the optimal usage strategy offers an efficient and integral way of preserving energy just by applying smart local algorithms on wireless units. In addition, even with the use of energy harvesting or wireless power, it is still necessary to manage the power consumption, and therefore optimal usage strategies are crucial.

The basic idea in the optimal usage is utilizing the on-board computational capability of wireless sensors, aiming to minimize the required time and energy for estimating the state of the structure from the measured data. In a majority of WSN deployments for SHM, the network architecture is designed such that all data is sent to a central station for further data processing. In such a central data processing scheme, a long time and a large portion of the finite power resource is spent on wireless communication for transmission of collected data. However, through the use of an optimal usage strategy, a system can be designed such that it incorporates on-board computation and preserves time and power by avoiding the communication of the entire collected data.

Because the transmission of raw data is time-consuming and a wasteful use of energy resources, data compression (Lynch et al. 2003, Sadhu et al. 2012) and data conversion approaches, such as the use of frequency responses (Caffrey et al. 2004), have been developed to reduce the volume of communication. Research has also been focused on distributing damage detection algorithms to make them suitable for implementation on WSN (Lynch et al. 2004b, Gao et al. 2006, Hackmann et al. 2008). More recently, further progress has been made toward the concept of local data processing for modal identification such that just an informative and condensed format of data is communicated through the network. One example of distributed modal identification approaches is the use of regularized autoregressive (AR) models (Pakzad et al. 2011). This approach proposes a restriction on AR parameters of the multivariate AR models that eliminates the computation of correlation between signals measured at nodes that are far apart, thus reducing the volume of data passed through the network. This approach is shown to result in an $85 \%$ savings in communication load in some cases (Pakzad et al. 2011). Another example is coordinated computing strategy (Nagayama and Spencer 2007, Sim et al. 2009), which divides the network into a number of subnetworks with cluster heads in a hierarchical topology. In this design, all the leaf nodes in each subnetwork receive the data from cluster heads and estimate and broadcast the correlation functions (under the assumption of ambient excitation) for implementation of Eigensystem realization algorithm (ERA). Further improvement of the approach is the use of decentralized random decrement technique (Sim et al. 2010), in which instead of transmission of raw data from cluster head to leaf nodes, the trigger crossing information is sent for estimation of correlation functions. Through examples it is shown that broadcasting the trigger crossing information, instead of raw data, could reduce the communication burden by $20 \%-30 \%$. Although these approaches reduce the amount of communicated data, they are 
dependent on the network topology and restricted by underlying identification algorithms. Additionally, more algorithm improvement is still required to remedy the latency and power consumption issue that exists in current WSN deployments.

This paper presents a novel approach for modal identification of structural systems, suitable for addressing challenges in application of WSN in SHM. The proposed algorithm, called iterative modal identification (IMID), assigns a computational task of modal identification to each remote node and limits the data communication to transmission of only modal analysis results. An iterative algorithm is developed such that each sensing node in the network sufficiently influences the final estimated results. The necessary requirement for the implementation of the proposed algorithm is an initial estimate of the structural modal properties, which can be available for many structural systems. This paper presents the formulation of this method, and as a proof of concept, the algorithm is implemented on numerically simulated shear structures and two experimental models.

The organization of the rest of this paper is as follows: The next section presents the significance of distributed modal identification algorithms in preserving time and energy through a detailed discussion on energy consumption of different components of a sensing node in a network. Then the theory and methodology of the proposed algorithm is presented. Afterward, the implementation of the algorithm is discussed and the validation of the algorithm through the use of numerically simulated and experimental models is presented. Another section evaluates the enhancement in efficiency of the modal identification by the use of the proposed algorithm, and finally, the last section presents conclusions on the performance of the proposed algorithm and its potential in different aspects of SHM.

\section{SIGNIFICANCE OF THE DISTRIBUTED MODAL IDENTIFICATION ALGORITHMS}

Latency in the data collection and prohibitive power consumption, as major drawbacks in application of wireless sensor networks in structural health monitoring, need special considerations in the design of WSN architecture. In a long-term SHM scenario, the monitoring system is expected to be prepared for collecting data and also for processing the data and extracting important information about the state of the structure. Due to the limited bandwidth in wireless networks, transmitting large volumes of data can take a prohibitively long time. In a large-scale deployment, tens of megabytes (MBs) of data need to be transferred through the network while the bandwidth is strictly limited. In one of the large-scale deployments of WSN, Pakzad et al. (2008) reported an average bandwidth of 550 bytes per second in multihop data transmission, which resulted in about nine hours for transferring $20 \mathrm{MB}$ of data from 64 sensor nodes to the base station. Such a delay prevents the monitoring system from prompt response, which is particularly required in the event of an earthquake.

In addition to the latency caused by the data transmission, power consumption also limits the performance of WSNs in long-term monitoring. Different components of a wireless sensor unit for vibration monitoring (e.g., accelerometer, processor, and transceiver) consume different amounts of energy when performing different tasks. Figure 1 shows a unit of a wireless sensor platform with its different components: SHM-A sensor board (Rice and Spencer 2008) and Imote 2 processing board (Crossbow 2007). Figure 2 presents a graph showing the results of the power consumption measurement on this wireless sensor unit 


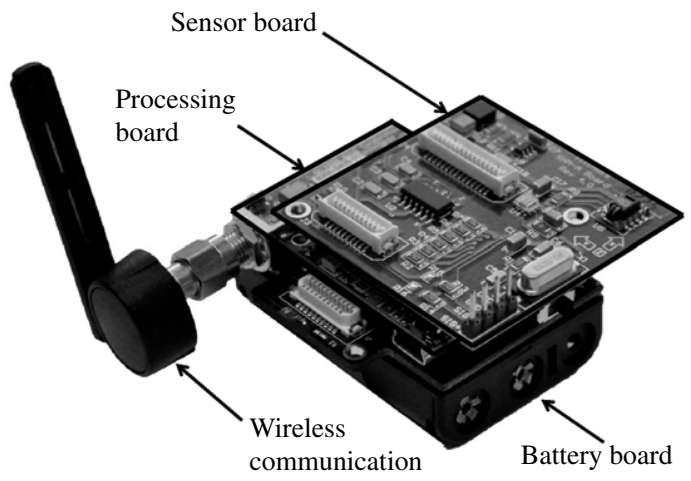

Figure 1. A wireless sensor platform (Imote2 and SHM-A).

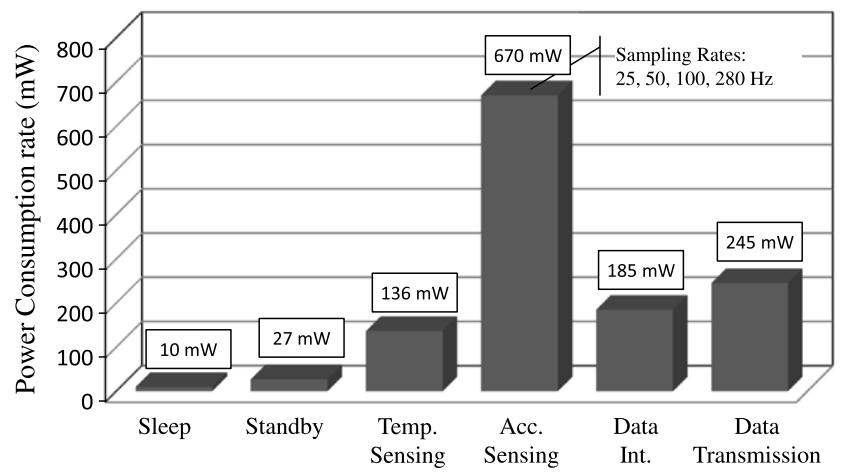

Figure 2. The power consumption rate during different tasks.

during the following tasks: standby, temperature sensing, acceleration sensing $(25,50,100$, and $280 \mathrm{~Hz}$ sampling rates), data interrogation, and data transmission. It is observed that the sampling rate in acceleration sensing has no effect on power consumption since it only depends on whether the sensor and ADC chips are powered on, and not on what task they are performing. This figure shows that the highest energy consumption rate corresponds to the acceleration sensing task and then the communication task. Considering the limited capacity of standard batteries and the fact that the battery voltage does not remain constant throughout usage, it is evident that a wireless node cannot last for long on its battery power.

Comparing the power consumption of different integrated components of a wireless sensor is important in managing the limited power resource. In an inclusive power consumption measurement, Whelan et al. (2009) reported a rate of $79.4 \mathrm{~mW}$ from all components of acceleration monitoring (for a specific wireless sensor platform) where $59 \mathrm{~mW}$ of that was from the CC2420 transceiver. Considering the communication time and the result of this power measurement, it is the radio transceiver that is the major power consumer in the entire unit. 
A distributed modal identification method incorporates the on-board processing capability to reduce the communication in the network. This approach is important because transmitting data over a wireless network not only takes a long time but also consumes significantly more energy than performing a local computation. An approximation of the required energy for performing a simple computation on one $\mathrm{kB}$ data and also for transmission of the same data through the radio signal can be obtained as:

$$
\begin{aligned}
E_{\text {computation }}= & {[\text { Number of cycles for computation }(\mathrm{ALU})] \times[1 / \text { Clock speed }(\mathrm{Hz})] } \\
& \times[\text { Power consumption rate }(\mathrm{mW})] \\
E_{\text {trsmissionn }}= & {[1 / \text { transmission rate }(\mathrm{Kbps})] \times[\text { Power consumption rate }(\mathrm{mW})] }
\end{aligned}
$$

where transmission rate, power consumption rate, and clock speed are specifications of the transceiver and the processor. The number of required cycles for computation depends on the algorithm that is used in the specific type of processor. ALU refers to arithmetic logic unit, which is a fundamental building block of a computer's CPU.

Considering the specifications of the CC2420 transceiver (Chipcon AS SmartRF 2004) and an Intel PXA27x processor, which are integrated into the Imote2 platform (Crossbow 2007), the estimated consumed energy for transmission of $1 \mathrm{kB}$ of data is $0.24 \mathrm{~mW} / \mathrm{sec}$, whereas the estimated required energy for a simple computation on $1 \mathrm{kB}$ of data is $2.25 \times$ $10^{-5} \mathrm{~mW} / \mathrm{sec}$. This result shows the noticeable higher power consumption of the transmission task and highlights the importance of developing and incorporating distributed modal identification algorithms to reduce the communication load and to preserve the limited energy.

\section{ITERATIVE MODAL IDENTIFICATION: THEORY AND METHODOLOGY}

A basic requirement for most of the existing modal identification algorithms is access to the entire measured data for computation of the full cross-correlation matrix. This restriction requires the sensor network to transmit all the collected data from each sensor to a base station. The proposed algorithm, called IMID, estimates the modal parameters of the system without requiring simultaneous access to the entire data.

IMID relies on a class of estimation algorithm called expectation-maximization (EM). EM estimates an unknown parameter $(\theta)$ given the measurement data $(Y)$ in the presence of some hidden variables $(\hat{Y})$ (Dempster 1977). This algorithm is in fact a generalized form of maximum-likelihood estimation, which is applicable when the data is incomplete. Considering the log-likelihood function of unknown parameters $\theta$ as:

$$
L(\theta)=\log [p(Y / \theta)]
$$

the estimation of unknowns $(\theta)$ is given by maximizing the function, $L(\theta)$, over $\theta$ :

$$
\theta=\operatorname{Arg} \cdot \max [L(\theta)]
$$

where $Y$ is the available data (complete measured data). 
When the entire data for estimation is not available (data is incomplete), an iterative method such as EM is applicable. EM first estimates the complete data using initially assumed parameters, $\theta^{p}$ (expectation phase), then maximizes the likelihood function over the system's parameter to find $\theta^{p+1}$ (maximization step) and continues until the convergence for the parameters is achieved. EM can be formally expressed as:

Expectation step:

$$
Q\left(\theta, \theta^{p}\right)=E\left[\log \left(p(X / \theta) / Y, \theta^{p}\right]\right.
$$

Maximization step:

$$
\theta^{p+1} \in \arg . \max Q\left(\theta, \theta^{p}\right)
$$

where $\theta^{p}$ denotes the value of the system's parameter obtained at the $p^{\text {th }}$ iteration. The concept of EM is also illustrated in Figure 3 (Moon 1996).

The similarity of the proposed identification algorithm (IMID) and EM is that in IMID, each node separately estimates the system's parameter $(\theta)$ based on the measured data at the node (available observation, $Y$ ) plus the assumed responses in the other nodes, $\hat{Y}$. In other words, each node estimates the system's parameter, having incomplete data.

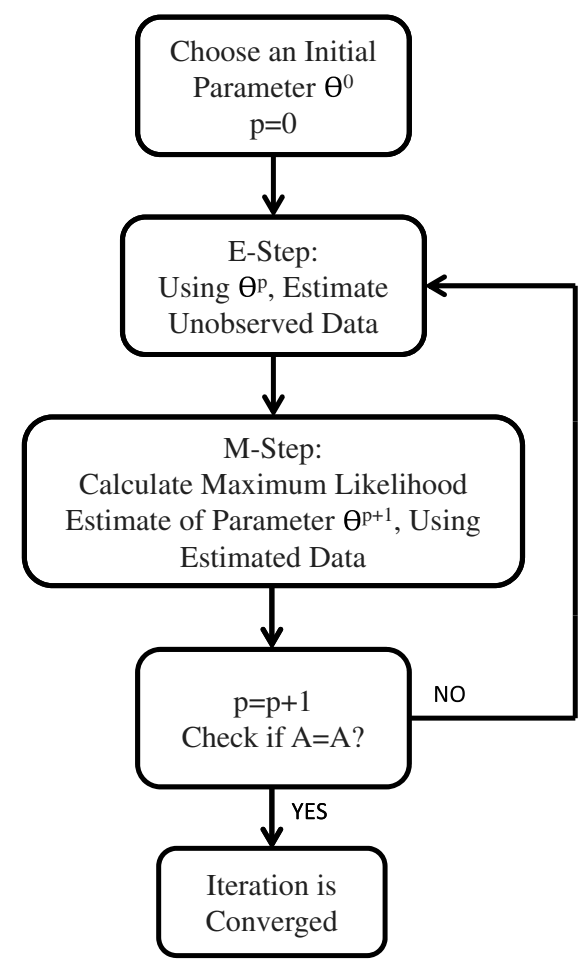

Figure 3. The block chart presenting the expectation-maximization (EM) algorithm. 
Considering a structure instrumented by $N$ sensor nodes, the first node uses the initial value of the parameters and makes a numerical simulation to obtain the response on other nodes (this step corresponds to the expectation step in EM algorithm). The simulated response and the measured data at this node are used to make an estimate on the system's modal parameters (this is the maximization step in EM algorithm). Then this node sends the system's parameters to the second node in the network for a similar local processing. These steps are taken in all of the nodes of the network one by one. However, for simulation of the response at other nodes, each node uses the system's parameter estimated at its previous node. Therefore, each node updates the estimation by the measured data at its location. This procedure is continued inside the network until the estimated parameters are stabilized and converged. Note that the proposed algorithm is developed for input-output systems and assumes that the excitation signal was measured and communicated through the network so all of the nodes have access to it. A possible scenario for application of input-output vibration testing is in the controlled load tests or in the impact test where the excitation is known to all the sensing nodes, as well as in earthquake events where the ground motion is also measured. Figure 4 shows a step-by-step block chart illustrating IMID.

Considering the fact that the transmission volume for the estimated parameters is very small compared to the time history data, IMID can significantly reduce the data traffic flowing through the network. As a result, it saves a considerable amount of time and energy in the communication task. It should be noted that implementation of the algorithm requires an initial estimate of the system's parameters required. This initial estimate can be achieved either from the finite-element model of the structure or, in long-term vibration monitoring,

1) Initially assumed Estimated system's parameters

2) Simulation of Response

3) Measurement of node's response

4) Combination of measured and simulated response

5) System identification

6) New estimated modal parameters

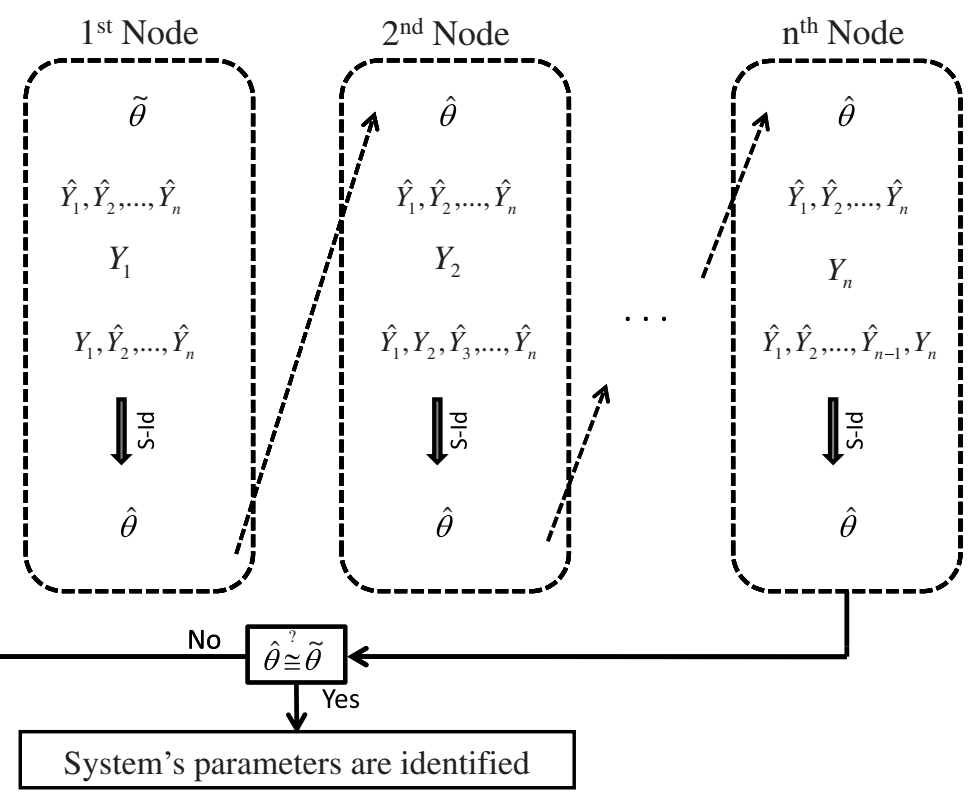

Figure 4. The block chart presenting the iterative modal identification (IMID) algorithm. 
from an initial data collection and centralized data processing. Considering that a large number of data sets are collected by permanent or semipermanent vibration monitoring systems (for example, 174 sets of data were collected in three months of monitoring the Golden Gate Bridge (Pakzad et. al. 2008) and more than 250 data sets were collected in about two months of vibration monitoring of Jindo Bridge (Jang et al. 2010)), and also the energy and time efficiency of the proposed method, it is reasonable to assume these initial estimates are available for sensing nodes in the beginning of the long-term monitoring program.

Fundamental iterative steps in IMID are (1) simulation of the response using the estimated parameters, and (2) modal identification using the collected data and simulated response. Several system identification models are available for both simulation of the response (e.g., Newmark numerical method and the use of autoregressive with exogenous (ARX) model) and modal realization (e.g., Eigensystem realization algorithm, stochastic subspace identification, ARX, and least-square algorithm). Selection of the best algorithm for each of these steps is one of the critical tasks in developing IMID. The performance of simulation and identification techniques, in terms of accuracy and efficiency, is the factor that needs to be considered in selection of algorithms for IMID. Additionally, since the objective of IMID is to utilize the on-board processors of the wireless sensors, it is also important to find a computationally efficient algorithm for implementation of IMID. The following section describes some implementation aspects of IMID algorithm.

\section{IMPLEMENTATION OF IMID}

As previously mentioned, in IMID all the nodes in the network contribute to the modal identification process. In order to explain the implementation details of the algorithm, the different tasks that need to be done in each node are discussed in this section. Figure 5 shows the necessary tasks that are assigned to each node in every iteration cycles.

\section{SIMULATION OF THE RESPONSE}

The first step in IMID is simulation of the dynamic response of the structure under the applied excitation. As long as the dynamic properties of the structure are available, this task can be done using any numerical methods. However, the selection of simulation method is related to the selected parameters for communication. For example, to apply numerical methods such as Newmark's method or central difference, the structural properties of the structure (i.e., mass, damping, and stiffness matrices) are required.

As an alternative method for simulation of response, the ARX model of the system can be used. In this approach, the response at current time can be estimated using ARX parameters,

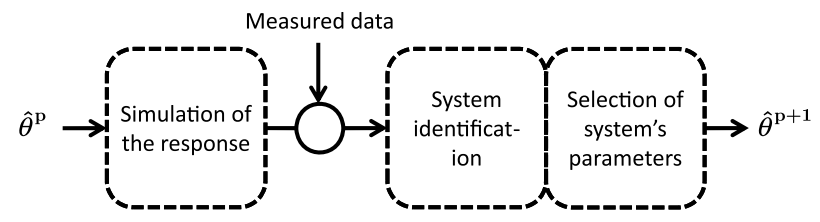

Figure 5. The block chart presenting the tasks assigned to each node. 
past response, and current and past inputs of the system. Utilizing ARX for simulation of the response requires ARX parameters to be transferred in the network.

\section{MODAL IDENTIFICATION}

After the dynamic response of the system is simulated at a remote node, the measured response is replaced with the corresponding simulated response at that point and, together with the simulated responses for other nodes, is fed into modal identification algorithm.

Consider the state-space representation of the dynamic system:

$$
\begin{gathered}
x(n+1)=A_{d} x(n)+B_{d} u(n), \\
y(n)=C x(n)+D u(n)
\end{gathered}
$$

where $x(n)$ is the state vector, $y(n)$ is the observation vector, and $u(n)$ is the input vector at time step $n ; A_{d}$ is the state and $B_{d}$ is input matrices in discrete format, $C$ is the observation matrix and $D$ is the transmission matrix. Eigenvalue decomposition of the state matrix $\left(A_{d}\right)$ results in the matrices of eigenvalues $\left(\lambda_{\mathrm{i}}{ }^{\prime} \mathrm{s}\right)$ and eigenvectors $\left(\psi_{\mathrm{i}}\right.$ 's) from which the natural frequencies, damping ratios, and mode shapes of the system can be obtained using the following relationships:

$$
\begin{gathered}
\lambda_{c_{i}}, \lambda_{c_{i}}^{*}=-\zeta_{i} \omega_{i} \pm j \omega_{i} \sqrt{1-\zeta_{i}^{2}}, \\
\phi_{i}=C \psi_{i},
\end{gathered}
$$

where $\omega_{i}$ and $\zeta_{i}$ are natural frequencies and damping ratios, respectively, and $\lambda_{c}$ is the eigenvalue of the continuous state matrix, which can be obtained from this equation:

$$
\lambda_{c_{i}}=\frac{\ln \left(\lambda_{i}\right)}{\Delta t}
$$

where $\Delta t$ is the sampling time. The goal in the identification step is to estimate the system (here, state-space matrices), which will result in accurate modal parameters.

\section{SELECTION AND ASSESSMENT OF THE SYSTEM'S PARAMETERS FOR TRANSMISSION}

Estimation of the system's parameters in IMID is not only the final objective but a step that is taken at each node in every iteration cycle. In fact, estimated parameters at each node are transmitted through the network to be updated node by node. Additionally, these parameters are checked for convergence at each cycle. Therefore, it is important to select the most informative parameters representing the system. Several alternatives for the system's parameters are ARX parameters, state-space matrices, and modal properties of the structure. 
The ARX parameters and state-space matrices can be the direct result of system identification step and no further assessment is needed before transmission of the parameters. However, extraction of structural properties (mass, damping, and stiffness matrices) from the result of the system identification step needs some considerations. It should be noted that the advantage of selecting these properties, compared to other alternatives, is their compact transmission volume.

Considering that the eigenvalues and eigenvectors of the system are estimated through the identification of the system's matrices, from the second order equation of motion, the eigenvalue equation can be written as:

$$
\left(\lambda_{n}^{2} m+\lambda_{n} c+k\right) \psi_{n}=0
$$

where $\lambda_{n}$ and $\psi_{n}$ are the $n^{\text {th }}$ complex eigenvalue and eigenvector of the dynamic system. Premultiplying this equation by $m^{-1}$, it can be rewritten as:

$$
\left[\begin{array}{ll}
m^{-1} k & \left.m^{-1} c\right]
\end{array}\left\{\begin{array}{c}
\psi_{n} \\
\lambda_{n} \psi_{n}
\end{array}\right\}=-\lambda_{n}^{2} \psi_{n}\right.
$$

which holds for each eigenvalue and eigenvector. Expanding the equation to include all $n$ modes of the system, it becomes:

$$
\left[\begin{array}{ll}
m^{-1} k & m^{-1} c
\end{array}\right]\left[\begin{array}{cccc}
\psi_{1} & \psi_{2} & \ldots & \psi_{n} \\
\lambda_{1} \psi_{1} & \lambda_{2} \psi_{2} & \ldots & \lambda_{n} \psi_{n}
\end{array}\right]=-\left[\begin{array}{llll}
\lambda_{1}^{2} \psi_{1} & \lambda_{2}^{2} \psi_{2} & \ldots & \lambda_{n}^{2} \psi_{n}
\end{array}\right]
$$

or

$$
\left[\begin{array}{ll}
m^{-1} k & m^{-1} c
\end{array}\right]=-\left[\begin{array}{llll}
\lambda_{1}^{2} \psi_{1} & \lambda_{2}^{2} \psi_{2} & \ldots & \lambda_{n}^{2} \psi_{n}
\end{array}\right]\left[\begin{array}{cccc}
\psi_{1} & \psi_{2} & \ldots & \psi_{n} \\
\lambda_{1} \psi_{1} & \lambda_{2} \psi_{2} & \ldots & \lambda_{n} \psi_{n}
\end{array}\right]^{*}
$$

where $*$ denotes the pseudo-inverse of a matrix. From this equation, $\left[\mathrm{m}^{-1} k\right]$ and $\left[\mathrm{m}^{-1} c\right]$ can be easily extracted with the minimum least-square error. Having an estimate on the mass of the system, stiffness and damping can be obtained.

The remaining issue is determination of eigenvalues and eigenvectors associated with the physical system from those generated by noise. During the system identification process, it is common to identify some modes that are not from the underlying physical system but are due to the noise in the data and overparameterization of the problem (computational and spurious modes). Constructing a stability diagram (Peeters and Roeck 2001) is an approach that addresses this difficulty based on the fact that structural modes stabilize through the process of increasing the model order whereas the spurious modes will not converge. Another available approach is determination of confidence interval for modes through the consistent mode indicator (CMI) (Pappa et al. 1997). In implementation of IMID, having an initial estimate of the system is also an advantage that can assist with determination of structural modes from all identified modes. 


\section{VALIDATION THROUGH NUMERICAL SIMULATION}

To validate the performance of IMID, it is implemented in the identification of two numerically simulated models. The models are a 5 degree of freedom (DOF) shear structure, shown in Figure 6a, and a 10 DOF mass-spring-damping model, shown in Figure 6b. The objective is to use IMID for identification of the modal properties of these systems. The simulation of the model and the steps of IMID are carried out in MATLAB (The Math Works, Inc. 1997).

As the starting point for the iteration process, stiffness matrices are perturbed in each case to form an initial estimate of the system. For perturbation, up to $20 \%$ random changes are applied to the stiffness coefficients and the perturbed coefficients are used as the initial estimate for the algorithm. Considering application of IMID in a long-term monitoring scenario, where the initial estimates can be obtained through an initial data collection and a centralized data processing, 20\% deviation of the initial estimate from the real values is substantial and larger than the deviation reported in long-term monitoring studies in literature as a result of environmental variation, measurement noise, or even small structural damage (e.g., statistical study performed on the 174 data sets collected during three months from the Golden Gate Bridge (Pakzad et al. 2008) concluded that the deviation in the estimated frequencies is at most $1.2 \%$ due to measurement noise and environmental effects). Also, considering the use of finite-element models for obtaining the initial estimate, the level of perturbation is consistent with practical scenarios, presented in the literature in the finite-element model updating field (Zhang et al. 2001, Brownjohn and Xia 2000).

In these numerically simulated examples, measurement noise is also added to the responses to verify the functionality of the algorithm in the presence of noises. The noise signals have a root mean square (RMS) equal to $10 \%$ of the response signal's RMS. The mass and damping ratios are assumed to be constant throughout the iterations and stiffness coefficients are selected for updating and transmission through the network.

\section{SIMULATION STEP}

For simulation of the response, Newmark's numerical method is used. This numerical time-stepping method calculates the response of a structural system under any arbitrary excitation. Through the use of Newmark's method, the response at any time step is calculated based on the response at the previous time step and the applied force.

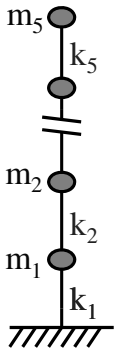

(a)

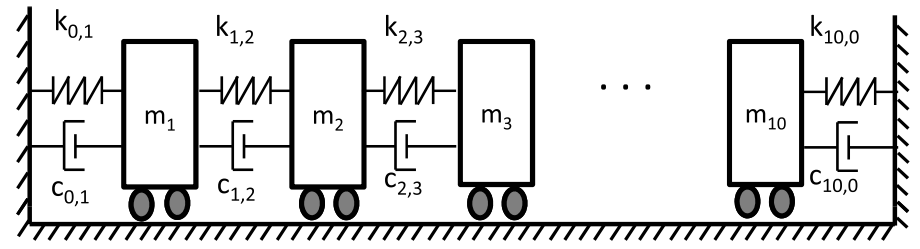

(b)

Figure 6. The (a) 5 DOF and (b) 10 DOF numerically simulated models. 


\section{IDENTIFICATION STEP}

In order to estimate the system's parameters, an ARX algorithm (Pandit 1991, Roeck et al. 1995) is used. The ARX model can be written as:

$$
\sum_{i=0}^{p} \alpha_{i} y(n-i)=\sum_{i=1}^{q} \beta_{i} x(n-i)+e(n)
$$

where $y=\left[\begin{array}{llll}y_{1}(n) & y_{2}(n) \ldots y_{m}(n)\end{array}\right]$ and $x(n)=\left[\begin{array}{llll}x_{1}(n) \mathrm{x}_{2}(n) \ldots x_{r}(n) & \ldots\end{array}\right.$ are matrices including output and input vectors respectively, $\alpha_{i}$ 's and $\beta_{i}$ 's are ARX coefficients, $e(n)$ represents the noise and measurement error, and $p$ and $q$ are orders of the autoregressive and exogenous parts of the ARX model that are assumed to be equal.

Having the AR parameters of the system, the state matrix can be expressed in the controller form as:

$$
A=\left[\begin{array}{cccccc}
0 & I & . & . & 0 & 0 \\
0 & 0 & . & . & 0 & 0 \\
. & . & . & . & . & . \\
. & . & . & . & . & . \\
0 & 0 & . & . & 0 & I \\
-\alpha_{p} & -\alpha_{p-1} & . & . & . & -\alpha_{1}
\end{array}\right]
$$

and also the observation matrix can be formed as:

$$
C=\left[\begin{array}{llllll}
I & 0 & . & . & 0 & 0
\end{array}\right]
$$

where $[I]$ and $[0]$ are identity and zero matrices with appropriate dimensions, respectively. From the state and observation matrices, modal properties of the system can be obtained using Equations 9-11.

\section{RESULTS OF THE IMPLEMENTATION}

To check the convergence of the results, the stiffness coefficients of the structure, identified frequencies, and mode shapes are assessed after each iteration cycle. The criterion for convergence of modal properties is defined by a predetermined threshold for the estimated parameters of the system at the end of each cycle. Modal accuracy criterion (MAC) value is used for comparison of mode shapes in consecutive cycles. This criterion is defined as:

$$
M A C=\frac{\left(\phi_{p}^{T} \times \phi_{p+1}\right)^{2}}{\left(\phi_{p}^{T} \times \phi_{p}\right) \times\left(\phi_{p+1}^{T} \times \phi_{p+1}\right)}
$$

where $\phi_{p}$ and $\phi_{p+1}$ are the estimated mode shapes at iteration cycles $p$ and $p+1$. 
The error percentages of stiffness coefficients, natural frequencies, and mode shapes in the initial estimates and during the iterations are presented in Figure 7 and Figure 8 for 5 and 10 DOF models, respectively. Depending on the desired level of accuracy, consistent results are achieved in all of the cases presented in the simulated examples. The identified modal
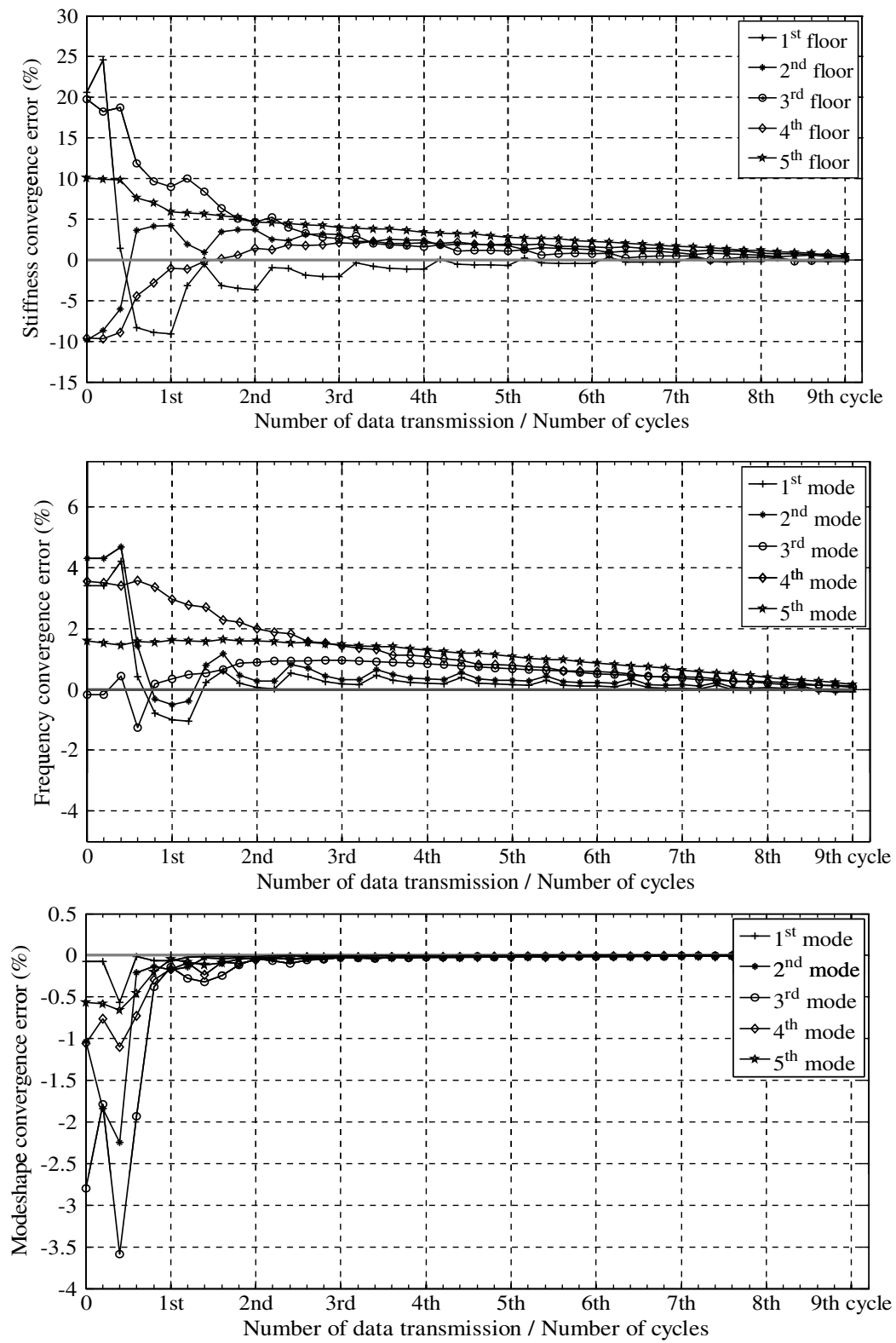

Figure 7. (a) to (c), the convergence of stiffness coefficients and modal properties of the 5 DOF model. 

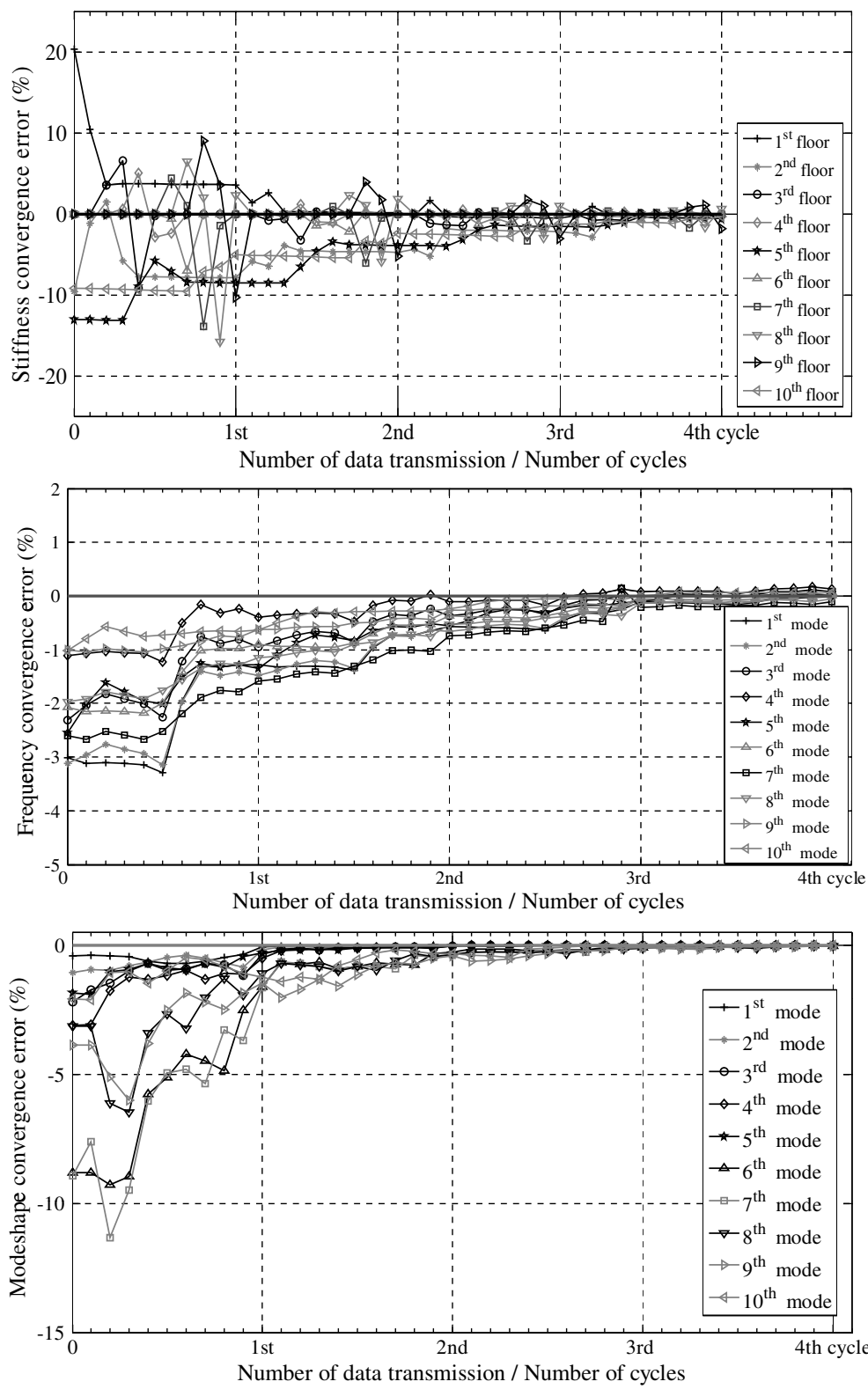

Figure 8. (a) to (c), the convergence of stiffness coefficients and modal properties of the 10 DOF model.

properties of the two models (5 and $10 \mathrm{DOF}$ ), from the finite-element model, IMID, and centralized ARX technique, are also presented in Table 1 and Table 2.

To identify the sensitivity of the convergence to the level of initial estimate error, a large set of simulations are performed with different levels of initial perturbations for the 5 DOF 
Table 1. Comparison of identified modal properties of 5 DOF model, using different algorithms

\begin{tabular}{|c|c|c|c|c|c|c|c|}
\hline \multicolumn{3}{|c|}{ Frequencies $(\mathrm{Hz})$} & \multicolumn{2}{|c|}{ MAC value } & \multicolumn{3}{|c|}{ Damping ratio (\%) } \\
\hline $\begin{array}{l}\text { True value } \\
\text { (FE model) }\end{array}$ & $\begin{array}{c}\text { Centralized } \\
\text { ARX }\end{array}$ & $\begin{array}{l}\text { IMID } \\
\text { after } 7 \\
\text { cycles }\end{array}$ & $\begin{array}{l}\text { FE vs. ARX } \\
\text { (centralized) }\end{array}$ & $\begin{array}{l}\text { FE vs. IMID } \\
\text { after } 7 \text { cycles }\end{array}$ & $\begin{array}{l}\text { True value } \\
\text { (FE model) }\end{array}$ & $\begin{array}{c}\text { Centralized } \\
\text { ARX }\end{array}$ & $\begin{array}{l}\text { IMID } \\
\text { after } 7 \\
\text { cycles }\end{array}$ \\
\hline 0.4158 & 0.4165 & 0.4153 & 1.0000 & 1.0000 & 5.000 & 5.000 & 5.042 \\
\hline 1.0197 & 1.0249 & 1.0184 & 1.0000 & 1.0000 & 5.000 & 5.000 & 5.031 \\
\hline 1.5724 & 1.5878 & 1.5715 & 1.0000 & 0.9996 & 5.000 & 4.990 & 4.985 \\
\hline 1.9449 & 1.9598 & 1.9444 & 1.0000 & 0.9996 & 5.000 & 4.980 & 4.966 \\
\hline 2.4391 & 2.4495 & 2.4385 & 1.0000 & 0.9997 & 5.000 & 4.970 & 4.964 \\
\hline
\end{tabular}

Table 2. Comparison of identified modal properties of 10 DOF model, using different algorithms

\begin{tabular}{|c|c|c|c|c|c|c|c|}
\hline \multicolumn{3}{|c|}{ Frequencies (Hz) } & \multicolumn{2}{|c|}{ MAC value } & \multicolumn{3}{|c|}{ Damping ratio (\%) } \\
\hline $\begin{array}{l}\text { True value } \\
\text { (FE model) }\end{array}$ & $\begin{array}{c}\text { Centralized } \\
\text { ARX }\end{array}$ & $\begin{array}{l}\text { IMID } \\
\text { after } 4 \\
\text { cycles }\end{array}$ & $\begin{array}{l}\text { FE vs. ARX } \\
\text { (centralized) }\end{array}$ & $\begin{array}{l}\text { FE vs. IMID } \\
\text { after } 4 \text { cycles }\end{array}$ & $\begin{array}{l}\text { True value } \\
\text { (FE model) }\end{array}$ & $\begin{array}{c}\text { Centralized } \\
\text { ARX }\end{array}$ & $\begin{array}{l}\text { IMID } \\
\text { after } 4 \\
\text { cycles }\end{array}$ \\
\hline 0.3141 & 0.3152 & 0.3132 & 1.0000 & 1.0000 & 5.0000 & 5.0153 & 5.0194 \\
\hline 0.5386 & 0.5428 & 0.5369 & 1.0000 & 1.0000 & 5.0000 & 5.0154 & 5.0288 \\
\hline 0.8206 & 0.8258 & 0.8190 & 1.0000 & 0.9999 & 5.0000 & 5.0090 & 5.0120 \\
\hline 1.0314 & 1.0436 & 1.0302 & 1.0000 & 0.9994 & 5.0000 & 5.0045 & 5.0110 \\
\hline 1.2685 & 1.2846 & 1.2653 & 1.0000 & 0.9994 & 5.0000 & 5.0108 & 5.0122 \\
\hline 1.5202 & 1.5395 & 1.5165 & 0.9998 & 0.9986 & 5.0000 & 5.0094 & 5.0109 \\
\hline 1.6378 & 1.6725 & 1.6316 & 0.9997 & 0.9932 & 5.0000 & 5.0160 & 5.0175 \\
\hline 1.7819 & 1.7612 & 1.7765 & 0.9997 & 0.9918 & 5.0000 & 5.0114 & 5.0224 \\
\hline 1.8672 & 1.8607 & 1.8633 & 0.9996 & 0.9973 & 5.0000 & 5.0060 & 5.0255 \\
\hline 1.9262 & 1.9259 & 1.9229 & 0.9998 & 0.9981 & 5.0000 & 5.0039 & 5.0124 \\
\hline
\end{tabular}

shear model. The initial estimates of stiffness components are selected to be ranged from $50 \%$ to $150 \%$ of the actual values, with a $5 \%$ increment. The results showed that, when the initial estimate error is from $5 \%$ to $25 \%$ (initial values are from $75 \%$ to $125 \%$ of the actual values), the convergence, with less than 1\% error, happens in less than eight cycles (increasing by the increase of initial estimate error). Also for initial estimate errors of $25 \%$ to $35 \%$, the convergence eventually happened after a maximum of 15 cycles. However, for initial estimate errors, beyond $35 \%$ in some cases, the algorithm did not converge. It should be noted that this level of error is beyond what is expected in application scenarios, discussed in the beginning of this section. 


\section{EXPERIMENTAL VALIDATION OF IMID}

The simulation results in the previous section showed the robustness of IMID. The next step is experimental validation of the algorithm. For this purpose, a series of dynamic tests were performed on two experimental structures in the laboratory. The first one is a five-story model structure excited with a shaking table (Figure 9a), and the second is a truss structure excited by impulsive load, using a hammer test (Figure 9b). The implementation on the fivestory model is presented with details in this paper. However, for the truss structure, only the results and a brief discussion are presented. For more information about the details of this experiment, the reader is referred to Dorvash and Pakzad (2012).

\section{FIVE-STORY MODEL STRUCTURE}

The structure is a five-story laboratory model with 5 degrees of freedom and is subjected to earthquake ground excitation. The excitation is from the 1940 El Centro, California, earthquake record ( $\mathrm{S} 00 \mathrm{E})$, and the response is recorded via five Imote2 wireless sensors with SHM-A sensor boards using $280 \mathrm{~Hz}$ sampling rate. The framework for programming Imote2 is TinyOS operating system. For collecting data, a software package developed by Illinois Structural Health Monitoring Project (ISHMP 2009) is used. As an essential requirement in vibration monitoring using wireless sensors, time synchronization is utilized in an acceleration-sensing application of the ISHMP tool suite. Time synchronization of sensing nodes is a relatively short task (less than 30 seconds in this experiment) that is necessary in extraction of accurate mode shapes from modal identification of structural systems. The utilized time synchronization protocol in this application is TPSN (Ganeriwal et al. 2003).

The objective of the test is to use IMID to find the modal properties of the model. To have a basis for comparison of the results, the modal properties are identified using the stochastic subspace system identification (SSI) method (Overschee and Moore 1994) with a centralized data collection scheme. The estimated modal properties are presented in Figure 10. To begin the iteration, an initial estimate of the structural model is required as the starting point. For this example, masses are added to different floors, a separate modal analysis is conducted, and the identified parameters of the altered model are used as the initial estimate for the algorithm. This is similar to the perturbation of stiffness matrix that was used in the simulation examples.
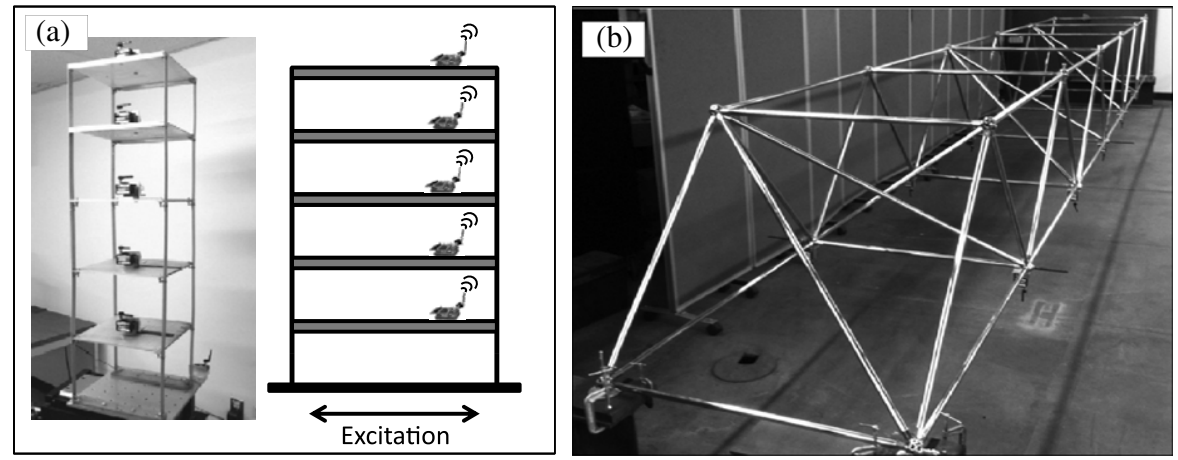

Figure 9. (a) The experimental model on shaking table; (b) the experimental truss in the laboratory. 

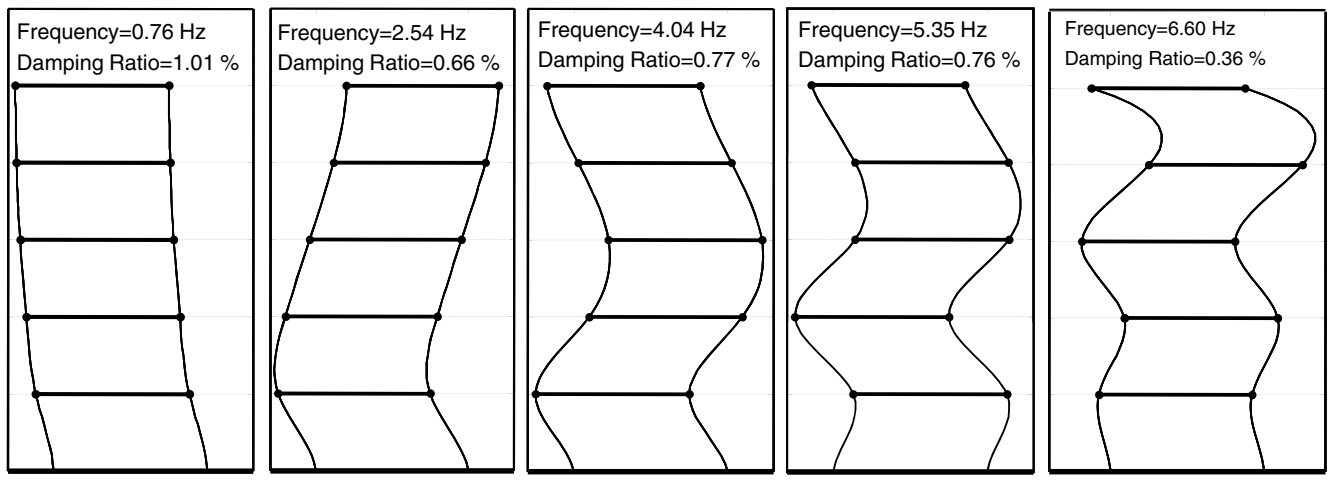

Figure 10. The identified modal properties of the experimental model.

Due to the contribution of disturbances in the measured data (e.g., measurement noise and nonlinearities in the response), careful considerations should be given to the selection of the model representing the structure. In contrast to the noise-free data where the structural properties $(m, c$, and $k)$ are sufficient to describe the system, in the experimental data more informative parameters are required to accurately characterize the underlying system. As an alternative to the structural properties, ARX parameters ( $\alpha_{i}$ 's and $\beta_{i}$ 's) can be used to represent the structural system in simulation. In this case, ARX parameters are considered to be transmitted through the network as the system's parameters. In comparison with structural properties, these parameters can carry a larger amount of information about the system. For example, a 5-degree-offreedom structure can be characterized by stiffness, mass, and damping matrices of size 5 by 5 . However, the ARX model with order 5 (for instance) describes the same system by 10 matrices of size 5 by 5 ( 5 autoregressive and 5 exogenous matrices). In the absence of noise, the larger size of these parameters does not provide any additional information for simulation. However, in practical problems, these parameters provide more accurate results. It should be noted that as the order of the assumed model increases, the size of the transmitted data increases and, consequently, the communication burden of the network also increases. Therefore, optimizing the selected model and the corresponding order is important. Additionally, high model order may result in an overparameterized model for the system and result in even less accurate estimation. In literature, there are established criteria that assist with finding the best order for systems (e.g., Akaike's information criterion (AIC), Brockwell and Davis 2002).

To illustrate the effect of model order in the accuracy of the simulation, the measured responses are compared to the simulation results, in different ARX model orders. Figure 11 shows the changes in the ratio of residual-to-response root mean square (RMS) versus the model orders. Residuals are defined as the difference of the simulated response and the measurement data. This ratio reflects the accuracy of the estimated model in terms of predicting the outputs. The results show that the optimum model order for this specific model is 13 . This optimum order is, of course, system and algorithm dependent, which means that for any different system, the best order should be obtained through a preprocessing. Figure 12 also shows the two simulated and measured responses when using the fitted model with 


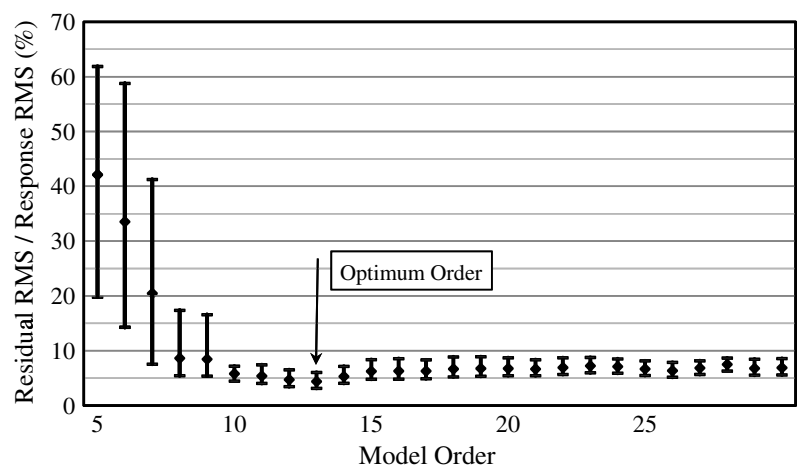

Figure 11. The residual-response ratio (maximum, minimum, and average of different nodes) versus model order.

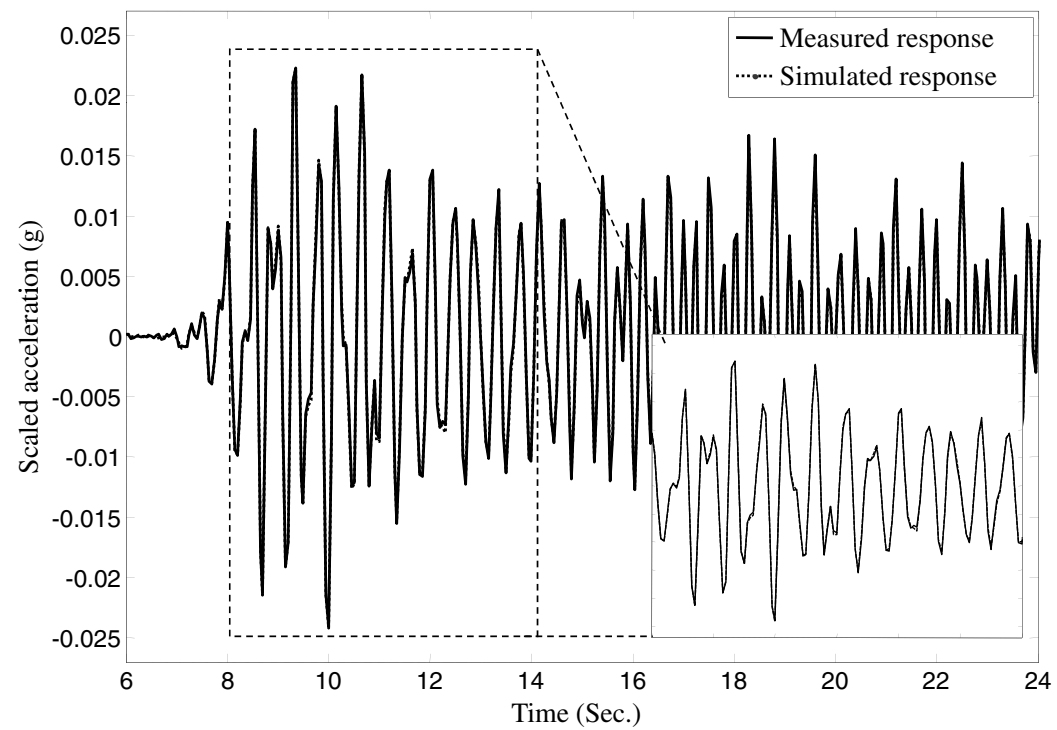

Figure 12. Measured response and simulated response from estimated model with optimal order.

selected order. Although the two signals are not perfectly identical, the differences are minimal when the proper model order is selected.

Once the order is selected, IMID starts iteration from an initial estimate using the ARX model for both simulation and identification. To track the convergence, the changes in the estimated modal properties are assessed at the end of each cycle. Figures $13 \mathrm{a}$ and $13 \mathrm{~b}$ show the error percentage in estimated natural frequencies and mode shapes versus the number of iteration cycles. It is shown that after a few cycles, modal properties converge with less than $1 \%$ error. The results are also compared to the modal properties that are extracted from 

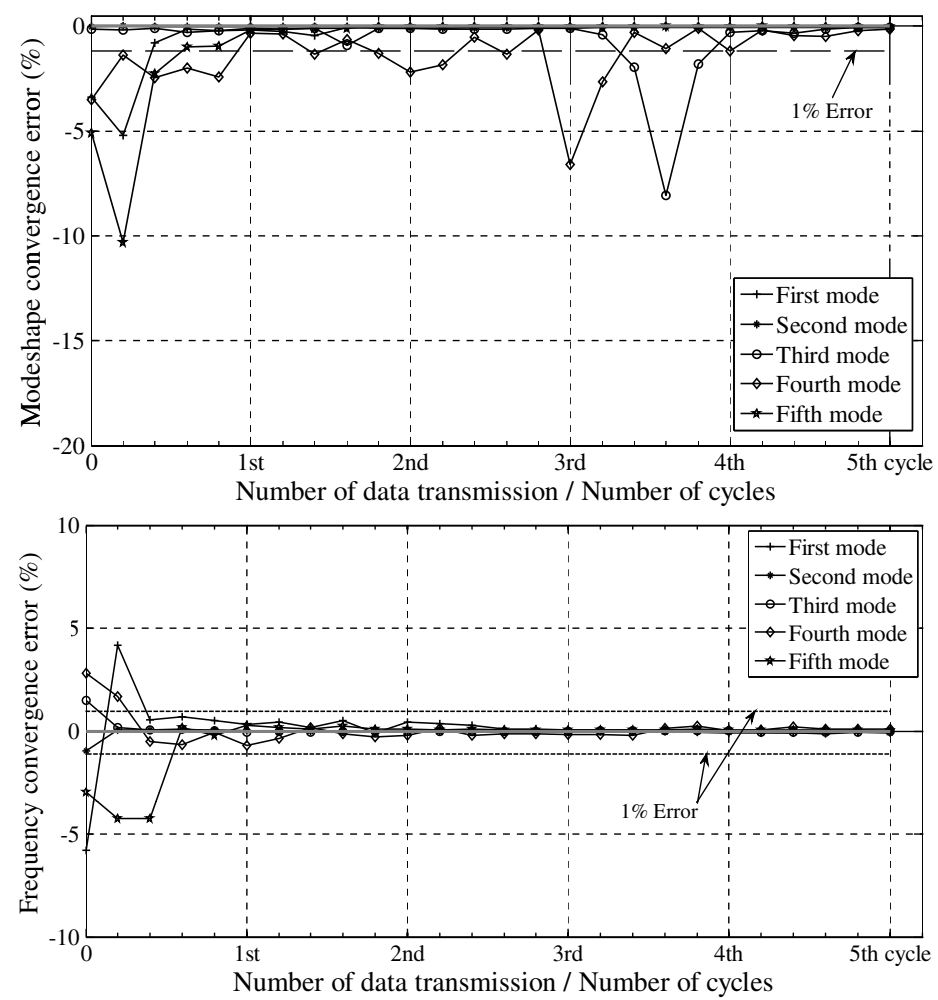

Figure 13. (a) and (b), the convergence of natural frequencies and mode shapes of five-story experimental model versus iteration cycles.

Table 3. Comparison of identified modal properties of the experimental model, using different algorithms

\begin{tabular}{|c|c|c|c|c|c|c|}
\hline \multicolumn{3}{|c|}{ Frequencies $(\mathrm{Hz})$} & \multicolumn{3}{|c|}{ Damping ratios (\%) } & \multirow{2}{*}{$\begin{array}{c}\text { MAC } \\
\text { IMID vs. SSI } \\
\text { after } 4 \text { cycles }\end{array}$} \\
\hline $\begin{array}{c}\text { Centralized } \\
\text { SSI }\end{array}$ & $\begin{array}{c}\text { Centralized } \\
\text { ARX }\end{array}$ & $\begin{array}{c}\text { IMID } \\
\text { after } \\
4 \text { cycles }\end{array}$ & $\begin{array}{c}\text { Centralized } \\
\text { SSI }\end{array}$ & $\begin{array}{c}\text { Centralized } \\
\text { ARX }\end{array}$ & $\begin{array}{c}\text { IMID } \\
\text { after } \\
4 \text { cycles }\end{array}$ & \\
\hline 0.7598 & 0.7590 & 0.7601 & 1.0049 & 0.9401 & 1.0620 & 0.9996 \\
\hline 2.5426 & 2.5425 & 2.5423 & 0.6569 & 0.6580 & 0.6385 & 1.0000 \\
\hline 4.0427 & 4.0428 & 4.0413 & 0.7741 & 0.7907 & 0.8385 & 0.9993 \\
\hline 5.3453 & 5.3311 & 5.3447 & 0.7568 & 0.8284 & 1.0952 & 0.9925 \\
\hline 6.6048 & 6.6076 & 6.6084 & 0.3574 & 0.3628 & 0.3738 & 0.9999 \\
\hline
\end{tabular}

centralized modal identification with ARX and SSI methods. Table 3 summarizes the results obtained from different algorithms. This table shows the accuracy of the obtained results from IMID, based on the estimated results from centrally processed data. 


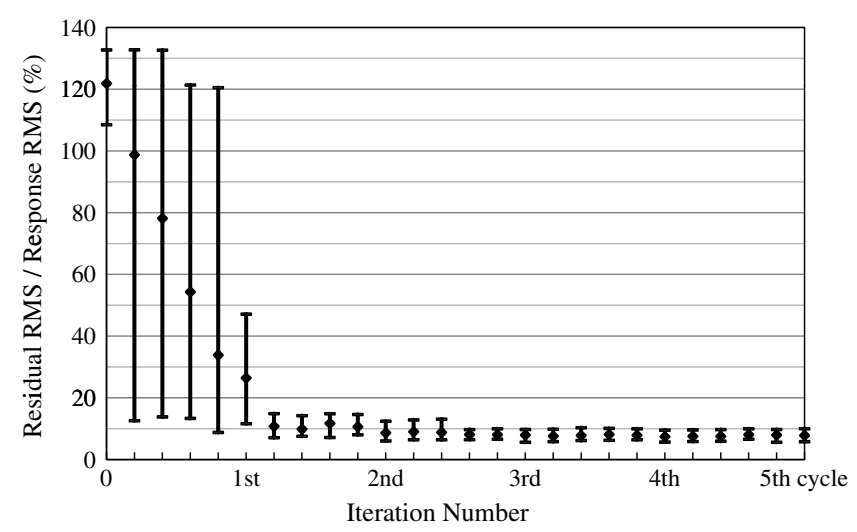

Figure 14. The residual-response ratio (maximum, minimum, and average of different nodes) versus iteration number.

Figure 14 also shows the changes in the ratio of residual-to-response RMS over the iteration cycles. This result supports the fact that the assumed model at different nodes of the network converges to the real system after a few cycles of iteration.

\section{EXPERIMENTAL TRUSS STRUCTURE}

To further validate the IMID, the algorithm is used for modal identification of a threedimensional steel truss structure. The truss is 27.5 feet long and has six panels, as shown in Figure 9b. A network of 10 wireless sensors is installed on the lower chord of the truss to collect the acceleration data in lateral and vertical directions. The structure is excited by impulsive load, using a hammer test.

The initially estimated model for iteration is formed by adding random masses to different nodes such that the additional masses make about a $10 \%$ change in the natural frequencies of the system. An autoregressive model is fitted to the response of the altered truss to represent the initial estimate of the system in the algorithm. The model order for this implementation is selected to be 10 , which results in 10 matrices of autoregressive parameters, communicated among the nodes of the network.

To check the convergence of the results, identified frequencies and mode shapes at each step are compared to those identified in the previous step and the error is calculated. Figures $15 \mathrm{a}$ and $15 \mathrm{~b}$ show the error percentage in estimated natural frequencies and mode shapes versus the iteration cycles. These figure show that before three cycles, the natural frequencies and mode shape errors are less than $2 \%$. More details about this experiment and the results can be found in Dorvash and Pakzad (2012).

\section{EFFICIENCY INVESTIGATION OF IMID}

The enhancement in modal identification, using the proposed algorithm, is evaluated in this section. The basis for this assessment is the reduction in the total communicated data points and also the total energy that is required for the estimation of structural modal properties. These parameters are selected since they reflect the improvement of modal identification 


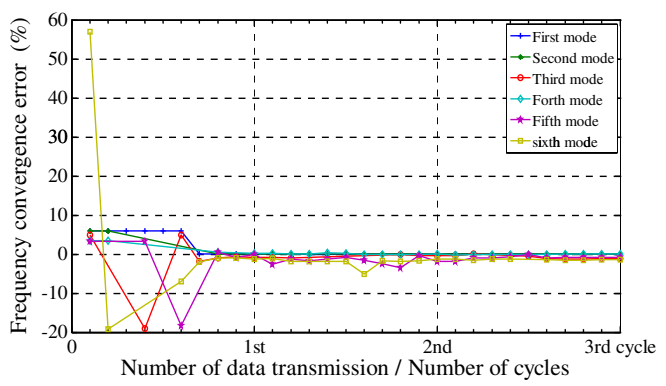

(a)

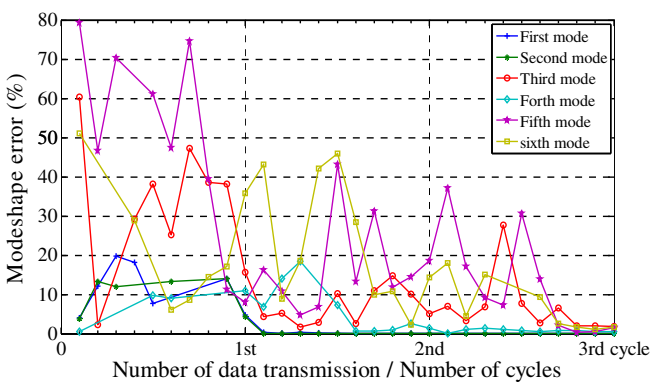

(b)

Figure 15. (a) and (b), the convergence of natural frequencies and mode shapes of the six identified modes of the experimental truss structure versus iteration cycles.

in terms of minimizing both latency and energy usage. The results of numerical examples and the experimental five-story model are used to evaluate the efficiency of IMID.

For the numerical example, the coefficients of stiffness matrix are transmitted through the network and therefore the total number of transmitted data points can be obtained from:

$$
\begin{aligned}
N_{\text {total }}= & (\text { number of cycles } \times \text { number of nodes }) \\
& \times(\text { number of stiffness matrix coefficients })
\end{aligned}
$$

Considering less than $1 \%$ error in convergence of modal parameters, the total number of transmitted data is:

$$
\begin{aligned}
& N_{\text {total }}=(7 \times 5) \times 5=175(\text { for } 5 \text { DOF model }) \\
& N_{\text {total }}=(4 \times 10) \times 10=400(\text { for } 10 \text { DOF model })
\end{aligned}
$$

For the experimental validation, the ARX parameters were sent through the network. In this case, based on the cycles of iteration and the selected ARX model, the total number of transmitted data points can be obtained as:

$$
\begin{aligned}
N_{\text {total }}= & (\text { number of cycles } \times \text { number of nodes }) \\
& \times(A R X \text { order } \times \text { size of } A R X \text { parameters })
\end{aligned}
$$

In the example of the five-story model, the number of nodes is five, the convergence with less than $1 \%$ error occurs after four cycles of iteration, and the selected ARX order is 10 . ARX parameters include two groups of autoregressive and exogenous parameters. The autoregressive parameters are each 5 by 5 whereas exogenous parameters can be vectors of size 5 . This is based on the fact that the excitations at different nodes are not independent and all are generated by the ground motion. Therefore, the total number of data points is equal to:

$$
N_{\text {total }}=(4 \times 5) \times[(10 \times 25)+(10 \times 5)]=6000(96 \mathrm{~kb})
$$




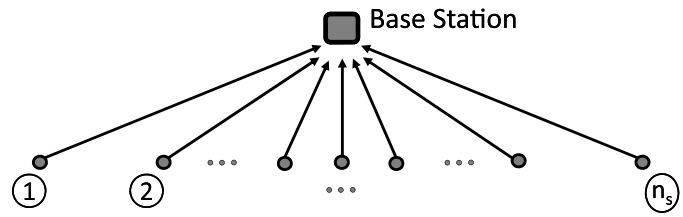

(a)

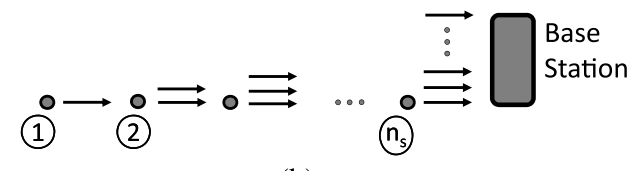

(b)

Figure 16. (a) The centralized data transmission routing; (b) multihop data transmission routing.

The efficiency of IMID becomes clearer when comparing this approach with the centralized computation method. For centralized computation, the data needs to be transferred to the base station. To transfer the collected data, sensors can either send their data directly to the base station (centralized data collection scheme, Figure 16a) or send them through the intermediary nodes, called multihop communication (Figure 16b). Use of multihop data transmission is essential when the network is long and there are nodes that are not in the radio range of the base station (e.g., implementation of WSN on long-span bridges). When using the centralized data transmission routing, the total number of data points will be simply $N . n_{s}$ where $n_{s}$ is the number of sensors and $N$ is the number of collected data points at each sensor. Also, in multihop communication routing, the total transmitted data is $N . n_{s}\left(n_{s}+1\right) / 2$ (Pakzad et al. 2008). In modal identification process, the higher the number of collected data samples is, the better the estimation of modal properties would be. In this implementation, 25,000 samples were collected at each sensor node. Considering use of centralized data transmission, 125,000 $(2,000 \mathrm{~kb})$ will be the total samples need to be communicated. In multihop data transmission and routing, which is essential in many wireless sensor network deployments, the significance of the proposed algorithm will be even more evident, as such implementations need $\left(n_{s}+1\right) / 2$ times larger transmission when collecting the data at the base station, but the same when using the IMID approach.

\section{COMPARISON OF THE TOTAL ENERGY}

Considering the results and specifications of the experimental implementation, the energy consumed by the two approaches, namely IMID and centralized system identification, are estimated and compared.

Required energy for data transmission can be calculated simply from Equation 2 by having the volume of the data, the transmission rate, and the power consumption rate:

$$
E_{\text {trsmission }}=[1 / \text { transmission rate }(\mathrm{Kbps})] \times[\text { power consumption rate }(\mathrm{mW})]
$$

where the nominal transmission rate of the embedded transceiver on Imote 2 is $250 \mathrm{kbps}$ (Crossbow 2007) and the power consumption rate, while transmitting the data, is $245 \mathrm{~mW}$ (Figure 2).

Estimating the required energy for data processing is more challenging since it needs an estimate of computation time. Chang and Pakzad (2012) conducted a comprehensive study on the required computational time for several different time domain system identification 
algorithms. In this study the required number of operations is presented as a function of model order, number of outputs, and signal length. Then the computation times in different algorithms are presented and compared. It is concluded through simulated examples that the total consumed time for performing the entire identification process for a system with 5 outputs and order of 10 is just a fraction of second (less than 0.1 second).

The computational time for the numerical simulation of the response, using an ARX model, is also measured for the 5-degree-of-freedom system of the presented example and resulted in less than 0.05 seconds of CPU time. Considering these estimations of computational time at each node, four cycles of iteration, and $184 \mathrm{~mW}$ power consumption rate (Figure 2) of the Imote2 in data processing mode (radio off), the consumed power for computation is about $400 \mathrm{~mW} / \mathrm{sec}$. Also, the required energy for transmission of $96 \mathrm{~kb}$ data, using Equation 2, will be about $94 \mathrm{~mW} / \mathrm{sec}$, which results in $494 \mathrm{~mW} / \mathrm{sec}$ total energy.

For application of the centralized data processing to this example, considering the transfer of 2,000 $\mathrm{kb}$ of data, the total required energy will be $1960 \mathrm{~mW} / \mathrm{sec}$.

This estimation shows a $75 \%$ savings in the consumed energy for IMID versus the centralized method. However, a few important considerations should be noted. First, the computational time may increase depending on the microcontroller that is responsible for on-board processing. If high performance processors are utilized in the next generations of wireless sensor units, this processing time can further decrease for the decentralize analysis. Second, the transmission rate considered in this study is the nominal rate presented in the data sheet of the CC2440 transceiver. However, in practice the actual transmission rates are less since there is a communication overhead due to packet loss and collisions in the wireless communication (e.g., see Nagayama et al. 2010, which reported a maximum 83 kbps communication rate in single-hop transmission in laboratory experiments). A lower communication rate causes a higher energy demand for the data transmission task, which adversely affects the centralized processing methods disproportionately. Third, in these estimations, it is assumed that the transmission scheme is centralized and single-hop. The use of multihop data transmission results in a longer data collection time. Therefore, the benefits of IMID will be more significant when a multihop data transmission scheme is utilized.

In comparing the required data transmission and energy consumption for IMID with those of centralized processing methods, it is evident that a substantial reduction in the network communication and the total consumed energy is achieved. Complete comparison with other decentralized system identification methods (e.g., Pakzad et al. 2011, Sim and Spencer 2009, Sim et al. 2010, Nagayama and Spencer 2007) may not be possible, since unlike those methods, IMID is an iterative process and the total computation/communication cost depends on how fast convergence could happen. The simulated and experimental results presented in this paper shows significant improvement in both agility and energy efficiency of the sensor network.

\section{CONCLUSION}

This paper presents a new distributed modal identification algorithm, called IMID, to address challenges in the application of wireless sensor networks in health monitoring of structural systems. The proposed algorithm is structured similar to iterative expectation-maximization series of algorithms that estimate unknown parameters in the presence 
of hidden information. The main objective in developing such an algorithm is to reduce the communication burden in WSN as a smart way of saving computation time and communication power. Latency and energy consumption are two factors that prevent WSNs from prompt response to earthquake events and also restrict their application in long-term monitoring. The proposed algorithm achieves significant reduction in the total transmitted data by incorporating the on-board computational capability of wireless sensors. To verify the performance of IMID, two numerically simulated models and two experimental models are used as the implementation test beds. The results illustrate the convergence of modal parameters after a few iteration cycles.

The advantage of IMID is its flexibility in collaboration with a broad range of identification and simulation algorithms. In this implementation, Newmark's numerical method and autoregressive with exogenous algorithms are used for simulation and identification steps. Further studies are required to evaluate the efficiency of IMID if other methods are used for these steps.

The application of this algorithm is particularly beneficial in long-term structural health monitoring in which the current state of the system can be considered as the initial estimate. These modal parameters are updated using data collected during the monitoring period. Having the identified modal properties of the system in a long period of time, applications such as damage detection and/or finite-element model updating can be utilized in the system as well.

\section{ACKNOWLEDGMENTS}

The research described in this paper is supported by the National Science Foundation through grant no. CMMI-0926898 by Sensors and Sensing Systems Program and by a grant from the Commonwealth of Pennsylvania, Department of Community and Economic Development, through the Pennsylvania Infrastructure Technology Alliance (PITA).

\section{REFERENCES}

Brockwell, P. J., and Davis, R. A., 2002. Introduction to Time Series and Forecasting, 2nd edition, Springer, New York.

Brownjohn, J. M. W., and Xia, P. Q., 2000. Dynamic assessment of curved cable-stayed bridge by model updating, Journal of Structural Engineering 126, 252-260.

Caffrey, J., Govindan, R., Johnson, E., Krishnamachari, B., Masri, S., Sukhatme, G., Chintalapudi, K., Dantu, K., Rangwala, S., Sridharan, A., Xu, N., and Zuniga, M., 2004. Networked sensing for structural health monitoring, Proceedings of the 4th International Workshop on Structural Control, New York, NY, 10-11 June, 57-66.

Chang, M., and Pakzad, S. N., 2012. Modified natural excitation technique for stochastic model identification, Journal of Structural Engineering, doi:10.1061/(ASCE)ST.1943541X.0000559.

Chipcon AS SmartRF®, 2004. CC2420 Preliminary Datasheet, rev 1.2.

Cho, S., Jo, H., Jang, S., Park, J., Jung, H.J., Yun, C.B., Spencer, Jr., B. F., and Seo, J., 2010. Structural health monitoring of a cable-stayed bridge using smart sensor technology: Data analysis, Smart Structures and Systems 6, 461-480.

Chung, H. C., Enotomo, T., Loh, K., and Shinozuka, M., 2004. Real-time Visualization of bridge structural response through wireless MEMS sensors, Proceedings of SPIE, Testing, 
Reliability, and Application of Micro- and Nano-Material Systems II, San Diego, CA, Vol. 5392, 239-246.

Crossbow, 2007. Imote2, high-performance wireless sensor network node, Product data sheet, http://www.xbow.com.

Das, N. K., Khorrami, F., and Nourbakhsh, S., 1998. A new integrated piezoelectric-dielectric microstrip antenna for dual wireless actuation and sensing functions, Proceedings of the SPIE, Smart Electronics and MEMS, San Diego, CA, Vol. 3328, 133-146.

Dempster, A. P., Laird, N. M., and Rubin, D. B., 1997. Maximum likelihood from incomplete data via the EM algorithm, Journal of Royal Statistical Society, Series B (Methodological), 39, $1-38$.

Dorvash, S., and Pakzad, S. N., 2012. Iterative modal identification algorithm: Implementation and evaluation, in Proceedings of the 30th International Modal Analysis Conference (IMAC $X X X)$, Jacksonville, FL, 30 January-2 February.

Ganeriwal, S., Kumar, R., and Srivastava, M. B., 2003. Timing-sync protocol for sensor networks. UC Los Angeles: Center for Embedded Network Sensing, http://escholarship.org/ $\mathrm{uc} /$ item $/ 5 \mathrm{mh} 7 \mathrm{~m} 01 \mathrm{j}$.

Gao, Y., Spencer, Jr., B. F., and Ruiz-Sandoval, M., 2006. Distributed computing strategy for structural health monitoring, Journal of Structural Control and Health Monitoring 13, 488-507.

Grisso, B. L., Martin, L. A., and Inman, D. J., 2005. A wireless active sensing system for impedance-based structural health monitoring, Proceedings of the 23rd International Modal Analysis Conference, Orlando, FL.

Hackmann, G., Sun, F., Castaneda, N., Chenyang Lu, and Dyke, S., 2008. A holistic approach to decentralized structural damage localization using wireless sensor networks, Real-Time Systems Symposium, Barcelona, Spain, 30 November-3 December, 35-46.

Illinois Structural Health Monitoring Project (ISHMP), 2009. ISHMP Services Toolsuite, http:// shm.cs.uiuc.edu/software.html.

Jang, S., Jo, H., Cho, S., Mechitov, K., Rice, J. A., Sim, S. H., Jung, H. J., Yun, C. B., Spencer, Jr., B. F., and Agha, G., 2010. Autonomous structural health monitoring using wireless smart sensors on a cable-stayed bridge, Proceedings of the Fifth International IABMAS Conference, Philadelphia, 11- July 2010, CRC Press.

Kim, J., Swartz, R. A., Lynch, J. P., Lee, J. J., and Lee, C. G., 2010. Rapid-to-Deploy reconfigurable wireless structural monitoring systems using extended-range wireless sensors, Smart Structures and Systems 6, 505-524.

Liu, S. C., and Tomizuka, M., 2003. Strategic research for sensors and smart structures technology, Proceedings of the International Conference on Structural Health Monitoring and Intelligent Infrastructure, Tokyo, Japan, 13-15 November, vol. 1, 113-117.

Lynch, J. P., Sundararajan, A., Law, K. H., Kiremidjian, A. S., and Carryer, E., 2003. Powerefficient data management for a wireless structural monitoring system, Proceedings of the 4th International Workshop on Structural Health Monitoring, Stanford, CA, 15-17 September, $1177-1184$.

Lynch, J. P., Law, K. H., Kiremidjian, A. S., Carryer, E., Farrar, C. R., Sohn, H., Allen, D. W., Nadler, B., and Wait, J. R., 2004a. Design and performance validation of a wireless sensing unit for structural monitoring application, Structural Engineering and Mechanics, An International Journal, 17, 393-408. 
Lynch, J. P., Sundararajan, A., Law, K. H., Kiremidjian, A. S., and Carryer, E., 2004b. Embedding damage detection algorithms in a wireless sensing unit for operational power efficiency, Smart Materials and Structures 13, 800-810.

Lynch, J. P., Wang, Y., Law, K. H., Yi, J-H., Lee, C. G., and Yun, C. B., 2005. Validation of a large-scale wireless structural monitoring system on the Geumdang Bridge, Proceedings of the International Conference on Safety and Structural Reliability (ICOSSAR), Rome, Italy, 19-23 June.

The Math Works, Inc., 1997. MATLAB and Simulink, Natick, MA.

Moon, T. K., 1996. The expectation maximization algorithm in signal processing, IEEE Signal Processing Magazine 13, 47-60. DOI 10.1109/79.543975.

Nagayama, T., Moinzadeh, P., Mechitov, K., Ushita, M., Makihata, N., Ieiri, M., Agha, G., Spencer, Jr., B. F., Fujino, Y., and Seo, J. W., 2010. Reliable multi-hop communication for structural health monitoring, Smart Structures and Systems 6, 481-504.

Nagayama, T., and Spencer, Jr., B. F., 2007. Structural health monitoring using smart sensors, Newmark Structural Engineering Laboratory (NSEL) Report Series No. 1, University of Illinois at Urbana-Champaign, Urbana, Illinois, http://hdl.handle.net/2142/3521.

Overschee, P. V., and Moor, B. D., 1994. N4SID: Subspace algorithms for the identification of combined deterministic-stochastic systems, Automatica 30, 75-93.

Pakzad, S. N., 2010. Development and deployment of large scale wireless sensor network on a long-span bridge, Smart Structures and Systems 6, 525-543.

Pakzad, S. N., Fenves, G. L., Kim, S., and Culler, D. E., 2008. Design and implementation of scalable wireless sensor network for structural monitoring, Journal of Infrastructure Systems 14, 89-101.

Pakzad, S. N., Rocha, G. V., and Yu, B., 2011. Distributed modal identification using restricted auto regressive models, International Journal of Systems Science 42, 1473-1489. doi: 10.1080/00207721.2011.563875.

Pandit, S. M., 1991. Modal and Spectrum Analysis: Data Dependent Systems in State Space, John Wiley and Sons.

Pappa, R. S., James, G. H., and Zimmerman, D. C., 1997. Autonomous modal identification of the space shuttle tail rudder, NASA Technical Memorandum, Hampton, VA, $12 \mathrm{pp}$.

Peeters, B., and Roeck, G. D., 2001. Stochastic system identification for operational modal analysis: A review, Journal of Dynamic Systems, Measurement, and Control 123, 659-667.

Rice, J. A., and Spencer, Jr., B. F., 2008. Structural health monitoring sensor development for the Imote2 platform, Proceedings of SPIE Smart Structures/NDE, San Diego, CA, doi:10.1117/ 12.776695

Roeck, G. D., Claesen, W., and Broeck, P. V. D., 1995. DDS-methodology applied to parameter identification in civil engineering structures, Proceedings of Vibration and Noise, Venice, Italy, 341-353.

Sadhu, A., Bo, H., and Narasimhan, S., 2012. Blind source separation towards decentralized modal identification using compressive sampling, in Proceeding of 11th International Conference on Information Science, Signal Processing and their Applications (ISSPA), 1147-1152.

Sim, S. H., Carbonell-Marquez, J., Spencer, Jr., B. F., and Jo, H., 2010. Decentralized random decrement technique for efficient data aggregation and system identification in wireless smart sensor networks, Probabilistic Engineering Mechanics 26, 81-91. 
Sim, S. H., and Spencer, B. F., 2009. Decentralized strategies for monitoring structures using wireless smart sensor networks, NSEL Report Series, http://hdl.handle.net/2142/14280.

Swartz, R. A., Jung, D., Lynch, J. P., Wang, Y., Shi, D., and Flynn, M. P., 2005. Design of a wireless sensor for scalable distributed in-network computation in a structural health monitoring system, Proceedings of the 5th International Workshop on Structural Health Monitoring, Stanford, CA.

Tanner, N. A., Wait, J. R., Farrar, C. R., and Sohn, H., 2003. Structural health monitoring using modular wireless sensors, Journal of Intelligent Material Systems and Structures 14, 43-56.

TinyOS, 2006. http://www.tinyos.net.

Whelan, M. J., and Janoyan, K. D., 2009. Design of a robust, high-rate wireless sensor network for static and dynamic structural monitoring, Journal of Intelligent Material Systems and Structures 20, 849-863.

Zhang, Q. W., Chang, T. Y. P., and Chang, C. C. 2001. Finite-element model updating for the Kap Shui Mun cable-stayed bridge, Journal of Bridge Engineering 6, 285-293.

(Received 1 August 2011; accepted 1 March 2012) 